\title{
Low KRT15 expression is associated with poor prognosis in patients with breast invasive carcinoma
}

\author{
PENGCHENG ZHONG, RONG SHU, HUIWEN WU, ZHIWEN LIU, XIAOLING SHEN and YINGJIE HU \\ Laboratory of Herbal Drug Discovery, Science and Technology Innovation Center, \\ Guangzhou University of Chinese Medicine, Guangzhou, Guangdong 510405, P.R. China
}

Received August 18, 2020; Accepted December 1, 2020

DOI: $10.3892 /$ etm.2021.9736

\begin{abstract}
Although keratin 15 (KRT15) has been indicated to be overexpressed in several types of tumor, its role in breast invasive carcinoma (BRCA) has so far remained elusive. The aim of the present study was to explore KRT15 expression in BRCA based on data obtained from The Cancer Genome Atlas and The Genotype-Tissue Expression. KRT15 expression was compared using a Wilcoxon rank-sum test. Functional enrichment analysis was performed to reveal the biological roles and pathways of KRT15. The association between KRT15 expression and immune-cell infiltration was evaluated via single-sample gene set enrichment analysis (ssGSEA). To investigate the relationship between clinicopathological features and KRT15 expression, the prognostic value of KRT15 and other clinical factors was evaluated using Cox regression analysis and Kaplan-Meier (KM) plots. Subgroup prognostic analysis was also performed using forest plots and KM curves. Finally, a tissue microarray was used to assess KRT15 expression in BRCA tissues. KRT15 expression was significantly lower in BRCA tissues compared with that in normal tissues. Functional enrichment analysis suggested that KRT15-related genes were primarily enriched in the transmembrane transporter complex, cornification and ligand-receptor interactions. Increased KRT15 was associated with several tumor-suppressive pathways. ssGSEA revealed that high KRT15 expression was significantly associated with natural killer-cell, B-cell and mast-cell infiltration. Significant associations were observed between low KRT15 expression and advanced stage clinicopathological factors, as well as unfavorable overall survival (OS) and disease-specific survival. Multivariate Cox regression analysis suggested that KRT15 was an independent prognostic factor for $\mathrm{OS}(\mathrm{P}=0.039$;
\end{abstract}

Correspondence to: Professor Yingjie Hu, Laboratory of Herbal Drug Discovery, Science and Technology Innovation Center, Guangzhou University of Chinese Medicine, 12 Jichang Road, Guangzhou, Guangdong 510405, P.R. China

E-mail: yingjiehu@gzucm.edu.cn

Key words: keratin 15, breast invasive carcinoma, immune cell infiltration, prognosis, bioinformatics hazard ratio, $0.590 ; 95 \% \mathrm{CI}, 0.358-0.974)$. Subgroup prognostic analysis demonstrated that low KRT15 was a reliable predictor of poor OS. Immunohistochemistry of a tissue microarray indicated that positive KRT15 expression rates were significantly higher in normal tissues compared with those in the BRCA tissues. In conclusion, low KRT15 expression was significantly associated with poor prognosis in patients with BRCA. Thus, KRT15 may serve an important role in BRCA progression and may be used as a promising prognostic marker for diagnostic and prognostic analyses in patients with BRCA.

\section{Introduction}

Breast cancer is the most commonly diagnosed malignant neoplasm and is the second most common cause of cancer-related death among females worldwide (1). Breast invasive carcinoma (BRCA) accounts for the largest proportion of breast cancer cases, exhibits high metastatic capacity and is associated with poor prognosis (2). Although notable improvements have been made in its diagnosis and treatment in recent years, several serious challenges remain, such as high heterogeneity, dormant micro-metastases and resistance to chemotherapy, which result in a high rate of morbidity and mortality (3-6). Therefore, novel prognostic markers and therapeutic targets for breast cancer treatment are urgently required. Owing to the development of sequencing technologies and free access to curated databases, such as The Cancer Genome Atlas (TCGA), novel biomarkers for tumor diagnosis, prognosis and therapeutic assessment are more readily identifiable (7).

Keratin 15 (KRT15), also known as cytokeratin 15, is located on chromosome 17q21.2 and is a member of the KRT gene family. KRT15 has previously been reported to be a marker for skin stem cells in the hair follicle bulge (8) and serves a critical role in epidermal homeostasis (9). KRT15 has recently been demonstrated to be closely associated with tumorigenesis. KRT15 overexpression was observed in squamous-cell carcinoma samples (10) and is related to a poor prognosis in colorectal cancer (11). In addition, stem cells with KRT15-positive surface markers may give rise to certain types of cancer under certain conditions $(12,13)$. However, the roles of KRT15 in BRCA have remained largely elusive.

In the present study, to elucidate the potential prognostic value of KRT15, RNA-sequencing (RNA-seq) data for BRCA 
and clinical patient data were downloaded from TCGA. These data were used for differential gene expression and functional enrichment analyses to identify the genes and functional pathways highly associated with KRT15 expression. Tumor immune infiltration analysis was then performed to explore the relevance of KRT15 expression regarding immune-cell infiltration. Furthermore, the association of KRT15 with demographic and clinicopathological parameters was assessed and survival and clinical subgroup prognostic analyses were performed to evaluate the prognostic value of KRT15 in BRCA. Finally, further biological verification was performed using microarray analysis of BRCA tissues. Through integrative bioinformatics and statistical analyses, it was demonstrated that KRT15 may serve as a novel biomarker for predicting the prognosis of patients with BRCA.

\section{Materials and methods}

Data sourcing and preprocessing. RNA-seq datasets from BRCA projects [level 3 HTSeq fragments per kilobase per million (FPKM)] were downloaded from TCGA (portal. gdc.cancer.gov) on April 12th, 2020. Samples without corresponding clinical information were excluded. The format of the RNA-seq data was converted from level 3 HTSeq-FPKM to transcripts per million reads (TPM). Individual unavailable or unknown clinical information was regarded as missing values. In addition, processed by the TOIL pipeline (14), TCGA and GTEx RNA-seq data in the TPM format were collected from the University of California Santa Cruz XENA browser (xenabrowser.net/datapages) to compare KRT15 expression between normal tissues and BRCA tissues, as well as to perform a pan-cancer analysis. Furthermore, receiver operating characteristic (ROC) curve analysis was performed to evaluate the efficiency of using KRT15 to distinguish tumor tissues from non-tumor tissues by using the pROC package (15).

Tumor samples were classified into the low-expression or high-expression group according to the median expression levels of KRT15. The clinical features included age, TNM stage, pathological stage, progesterone receptor (PR)/estrogen receptor (ER)/human epidermal growth factor receptor 2 (HER2) status and Prediction Analysis of Microarray 50 (PAM50) subtype. As the data sources and processes included in the present study did not involve human participants or animals, no ethical approval or informed consent was required.

Differentially expressed gene (DEG) analysis. To determine DEGs, the DESeq2 package (16) was used to compare expression profiles (HTSeq-counts) between high and low KRT15 expression groups, as aforementioned. $\log _{2}$ fold change $\left(\log _{2} \mathrm{FCl}\right)>1$ and an adjusted $\mathrm{P}$-value of $<0.05$ were used as the threshold for determining whether a gene was differentially expressed. The results of the DEG analysis were represented as a volcano plot and a heat map.

Functional enrichment analysis. In the present study, the $\mathrm{R}$ package clusterProfiler (17) was utilized to perform Gene Ontology (GO) term and Kyoto Encyclopedia of Genes and Genomes (KEGG) pathway enrichment analysis of DEGs between high and low KRT15 gene expression groups. GO terms in the three categories of biological process (BP), cellular component (CC) and molecular function (MF) were determined. An adjusted P-value of $<0.05$ was regarded as indicative of statistical significance.

Gene set enrichment analysis (GSEA). GSEA is a computational method using a gene expression matrix and different enriched signaling pathways to predict pathways and/or phenotypes related to genes (18). GSEA was performed using the gseKEGG function of the R ClusterProfiler package (version 3.6.0) (17). Genome permutations were performed 1,000 times for each analysis. C2: Curated gene sets (c2. cp.v7.0.symbols.gmt) in MSigDB Collections were selected as the reference for gene sets. A pathway term with $\mathrm{P}<0.05$ and false discovery rate (FDR) value $<0.25$ (or set normalized enrichment score $>1$ ) was considered to exhibit statistically significant enrichment.

Immune infiltration analysis by single-sample (ss)GSEA. ssGSEA was used to evaluate 24 types of immune cells that may infiltrate into the tumor immune microenvironment (19). ssGSEA scores were calculated using the GSVA Bioconductor package (20). The correlation between KRT15 and the relative abundance of 24 types of immune cells was analyzed using Spearman's rank correlation coefficient analysis. The immune infiltration scores were compared between the low and high KRT15 expression groups using the Wilcoxon rank-sum test.

Clinical association analysis of KRT15 expression in BRCA. All statistical analyses were performed in $\mathrm{R}$ version 3.6.2. The Wilcoxon rank-sum test and Wilcoxon signed-rank test were used to compare KRT15 expression between unpaired and paired groups, respectively. The Wilcoxon signed-rank test, Kruskal-Wallis test, $\chi^{2}$ test and logistic regression analysis were performed to determine relationships between clinicopathological features and KRT15 expression. Kaplan-Meier (KM) curves were generated using the 'survminer' $\mathrm{R}$ package (CRAN.R-project.org/package=survminer) to evaluate the prognostic value of various clinical features. Multivariate Cox regression models were used to compare the effects of KRT15 expression on patient outcomes together with other clinicopathological features. $\mathrm{P}<0.05$ (two-tailed) was considered to indicate statistical significance in all tests. The hazard ratio (HR) with $95 \%$ confidence intervals (CI) was estimated to assess risk factors.

Construction and evaluation of the nomogram. Multivariate survival analysis was performed using Cox regression analysis to determine independent prognostic factors. To demonstrate the survival probability of patients with BRCA, a nomogram was constructed using the rms R package (rdrr.io/cran/rms), followed by generating calibration plots to examine the prediction efficiency of this model. The primary endpoints were overall survival (OS) and disease-specific survival (DSS) and $\mathrm{P}<0.05$ was considered to indicate statistical significance.

Subgroup prognostic analysis. Subgroup analyses were performed to investigate the associations between different clinicopathological characteristics and prognosis. Separate 
Cox proportional hazards models were used for individual subgroups during subgroup analysis and visualized using forest plots. KM analysis of different subgroups was performed using the package 'survminer'. $\mathrm{P}<0.05$ (two-tailed) was considered to indicate statistical significance.

Immunohistochemistry (IHC). BRCA tissue microarrays were purchased from Shanghai Outdo Biotech. Co., Ltd. The paraffin-embedded tissue chip contained 45 BRCA tissues and 45 paired paracancerous tissues. For IHC analysis, paraffin sections were dewaxed and hydrated. Following $0.01 \mathrm{M}$ citric acid repair liquid $(\mathrm{pH}$ 6.0) being added to repair antigen at $100^{\circ} \mathrm{C}$ under high pressure, the sections were washed and submerged in $\mathrm{H}_{2} \mathrm{O}_{2}(3 \%)$ for 30 min to block endogenous peroxidase, followed by the addition of $10 \%$ goat serum (Wuhan Boster Biological Technology, Ltd.) to block nonspecific binding for $30 \mathrm{~min}$ at room temperature. The primary antibody, rabbit anti-KRT15 monoclonal antibody (cat. no. ab52816; 1:300 dilution; Abcam) was subsequently added, followed by incubation at $4^{\circ} \mathrm{C}$ overnight. The sections were then warmed to room temperature, washed three times with TBST and then incubated with the secondary goat anti-rabbit antibody (cat. no. ab205718; 1:20,000 dilution; Abcam) for $45 \mathrm{~min}$ at $37^{\circ} \mathrm{C}$. After three washes with TBST, the antibodies were visualized with diaminobenzidine and the sections were examined under a light microscope to observe any brown stain indicative of positivity. Finally, the sections were sealed with neutral balsam, dried and analyzed using a light microscope. KRT15 staining was assessed independently by two pathologists who were blinded to all clinical information. Staining intensity and the percentage of stained cells were used as indicators for semiquantitative evaluation, as described previously (21). These two scores were multiplied to obtain an IHC score for each case. A score of $<3$ was defined as low KRT15 expression and a score $\geq 3$ was defined as high KRT15 expression.

\section{Results}

KRT15 expression is low in patients with BRCA. In the present study, 1,065 samples were screened for subsequent analysis, including 111 matched cancer and paracancerous samples (Table I). The Wilcoxon rank-sum test was used to assess differential KRT15 expression between tumor samples and normal samples, as well as between tumor samples and paracancerous samples. As indicated in Fig. 1A, tumor samples exhibited lower KRT15 expression compared with normal samples $(\mathrm{P}<0.001)$. Similarly, KRT15 expression in paracancerous samples was higher than in tumor samples $(\mathrm{P}<0.001$; Fig. 1B). According to the results of the Wilcoxon signed-rank test, KRT15 was expressed at a lower level in tumor samples compared with that in the matched paracancerous samples $(\mathrm{P}<0.001$; Fig. S1A). In addition, KRT15 expression was compared between normal tissues from the GTEx plus TCGA databases and 33 types of cancer from TCGA using the Wilcoxon rank-sum test. KRT15 expression levels were significantly lower in the BRCA tissues compared with those in the normal tissues $(\mathrm{P}<0.001$; Fig. 1D). Similar results were obtained from the comparison between paracancerous samples and BRCA samples in the TCGA pan-cancer cohort. Likewise, compared with normal tissues, KRT15 expression was significantly decreased in adrenocortical carcinoma, diffuse Large B-cell Lymphoma, glioblastoma multiforme, head and neck squamous cell carcinoma, kidney chromophobe, acute myeloid leukemia, lower grade glioma, liver hepatocellular carcinoma, prostate adenocarcinoma, skin cutaneous melanoma and testicular germ cell tumors $(\mathrm{P}<0.001$; Fig. S1B). Furthermore, as presented in Fig. 1C, the area under the curve was 0.800 with a CI of $0.749-0.850$, indicating that KRT15 was able to efficiently distinguish BRCA from paracancerous tissues.

Functional enrichment of KRT15 in patients with BRCA. To determine whether KRT15 may serve a critical role in BRCA, differential expression analysis was performed by comparing gene expression profiles between low and high gene expression groups. Using the DESeq2 $\mathrm{R}$ package (adjusted $\mathrm{P}<0.05$ and $\mid \log \mathrm{FCl}>1.5), 942$ DEGs were identified (231 upregulated and 711 downregulated). The outcome of the differential expression analysis is presented as a volcano plot (Fig. 2A) and heatmap (Fig. 2B).

ClusterProfiler was used to perform GO functional enrichment analysis of KRT15-associated DEGs. The results of the GO analysis indicated that the BP and MF terms of KRT15-related genes included cornification, digestion, antimicrobial humoral response, feeding behavior, keratinization, receptor ligand activity, channel activity, transmembrane transporter activity and transmitter-gated ion channel activity (Fig. 2C and D). In terms of the CC category, the genes were primarily localized on the cell surface and were also present in components of the cellular membrane, such as in the gamma-aminobutyric acid receptor complex, dendrite membrane, ion channel complex, transmembrane transporter complex, transporter complex and neuron projection membrane (Fig. 2E). KEGG enrichment analysis suggested that neuroactive ligand-receptor interaction, protein digestion and absorption and nicotine addiction were significantly enriched (Fig. 2F).

Gene-related signaling pathways determined by GSEA. To identify the differential activation of signaling pathways in BRCA, GSEA was performed to uncover significant differences between the low and high KRT15 expression groups $(\mathrm{FDR}<0.25, \mathrm{P}<0.05)$ in an enrichment analysis of the MsigDB collection (set c2.cp). Amongst these, a total of 640 datasets met the aforementioned FDR criteria. As presented in Fig. 3A-G, seven pathways, including regulation of tumor protein (TP) 53 expression and degradation, the forkhead box (FOX)O pathway, $\triangle$ NP63 pathway, vitamin D receptor (VDR) pathways, the alternative reading frame (ARF) pathway, the caspase pathway and the IL27 pathway were significantly enriched in the KRT15 high expression group. Of note, two pathways, including the angiotensin converting enzyme 2 (ACE2) pathway and ghrelin pathway, were significantly enriched in the KRT15 low expression group (Fig. 3H-I), indicating KRT15 may be closely associated with these signaling pathways (Table SI).

KRT15 expression is correlated with immune-cell infiltration levels in BRCA. In the subsequent analyses, Spearman correlation analysis was performed to reveal the correlation 
Table I. Association between KRT15 expression and clinicopathological characteristics.

\begin{tabular}{|c|c|c|c|}
\hline Item & $\begin{array}{l}\text { Low expression of KRT15, } \\
n=533\end{array}$ & $\begin{array}{l}\text { High expression of KRT15, } \\
\qquad n=532\end{array}$ & P-value \\
\hline T stage & & & 0.096 \\
\hline $\mathrm{T} 1$ & $124(23.4)$ & $151(28.4)$ & \\
\hline $\mathrm{T} 2$ & $320(60.3)$ & $295(55.6)$ & \\
\hline T3 & $65(12.2)$ & 72 (13.6) & \\
\hline $\mathrm{T} 4$ & $22(4.1)$ & $13(2.4)$ & \\
\hline N stage & & & 0.794 \\
\hline N0 & $248(47.5)$ & $259(49.4)$ & \\
\hline N1 & $173(33.1)$ & $176(33.6)$ & \\
\hline $\mathrm{N} 2$ & $62(11.9)$ & $54(10.3)$ & \\
\hline N3 & $39(7.5)$ & $35(6.7)$ & \\
\hline M stage & & & 0.470 \\
\hline M0 & $438(97.3)$ & $451(98.3)$ & \\
\hline M1 & $12(2.7)$ & $8(1.7)$ & \\
\hline Pathologic stage & & & 0.652 \\
\hline I & 88 (16.9) & $92(17.6)$ & \\
\hline II & $297(57.1)$ & 309 (59.2) & \\
\hline III & $124(23.8)$ & $114(21.8)$ & \\
\hline IV & $11(2.1)$ & $7(1.3)$ & \\
\hline PR status & & & 0.004 \\
\hline Negative & $145(28.9)$ & $193(37.8)$ & \\
\hline Positive & $356(71.1)$ & $318(62.2)$ & \\
\hline ER status & & & $<0.001$ \\
\hline Negative & $80(15.9)$ & $157(30.7)$ & \\
\hline Positive & $423(84.1)$ & $355(69.3)$ & \\
\hline HER2 status & & & 0.005 \\
\hline Negative & $259(73.2)$ & $289(82.3)$ & \\
\hline Positive & $95(26.8)$ & $62(17.7)$ & \\
\hline PAM50 & & & $<0.001$ \\
\hline Basal & $45(8.4)$ & $145(27.3)$ & \\
\hline HER2 & $60(11.3)$ & $22(4.1)$ & \\
\hline LumA & $261(49.0)$ & $290(54.5)$ & \\
\hline LumB & $163(30.6)$ & $39(7.3)$ & \\
\hline Normal & $4(0.8)$ & $36(6.8)$ & \\
\hline Histological type & & & 0.002 \\
\hline Infiltrating ductal carcinoma & $393(83.3)$ & $364(74.7)$ & \\
\hline Infiltrating lobular carcinoma & 79 (16.7) & $123(25.3)$ & \\
\hline Ethnicity & & & 0.198 \\
\hline Asian & $34(7.3)$ & $26(5.1)$ & \\
\hline Black or African American & 78 (16.7) & $101(19.9)$ & \\
\hline White & $356(76.1)$ & $381(75.0)$ & \\
\hline Anatomic neoplasm subdivision & & & 0.928 \\
\hline Left & $278(52.2)$ & $275(51.7)$ & \\
\hline Right & $255(47.8)$ & $257(48.3)$ & \\
\hline TP53 status & & & 0.693 \\
\hline Mut & $169(34.3)$ & $166(35.8)$ & \\
\hline WT & $323(65.7)$ & $298(64.2)$ & \\
\hline PIK3CA status & & & 0.482 \\
\hline Mut & $156(31.7)$ & $158(34.1)$ & \\
\hline WT & $336(68.3)$ & $306(65.9)$ & \\
\hline
\end{tabular}


Table I. Continued.

Low expression of KRT15, $n=533$
High expression of KRT15, $\mathrm{n}=532$

P-value

Item

$59.00(48.00,69.00)$

$58.00(49.00,66.00)$

$0.377^{\mathrm{a}}$

Age, years

Values are expressed as n (\%) or median (interquartile range). ${ }^{a}$ Wilcoxon rank-sum test. KRT15, keratin 15; PR, progesterone receptor; ER, estrogen receptor; HER2, human epidermal growth factor receptor 2; PAM50, prediction analysis microarray 50; Lum, luminal; WT, wild-type; Mut, mutated; TP53, tumor protein 53; PIK3CA, phosphotidylinositol-4,5-bisphosphate 3-kinase catalytic subunit $\alpha$.

A

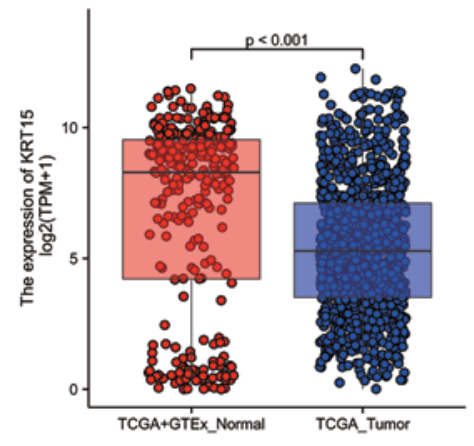

D
B

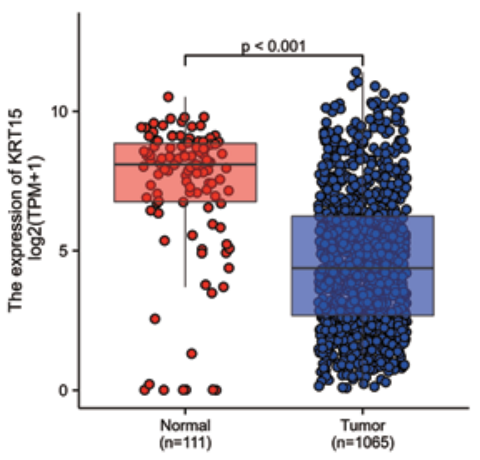

C

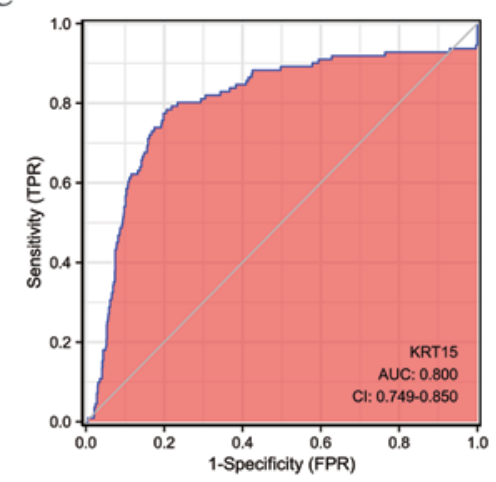

TCGA+GTEx_Normal TCGA_Tumor

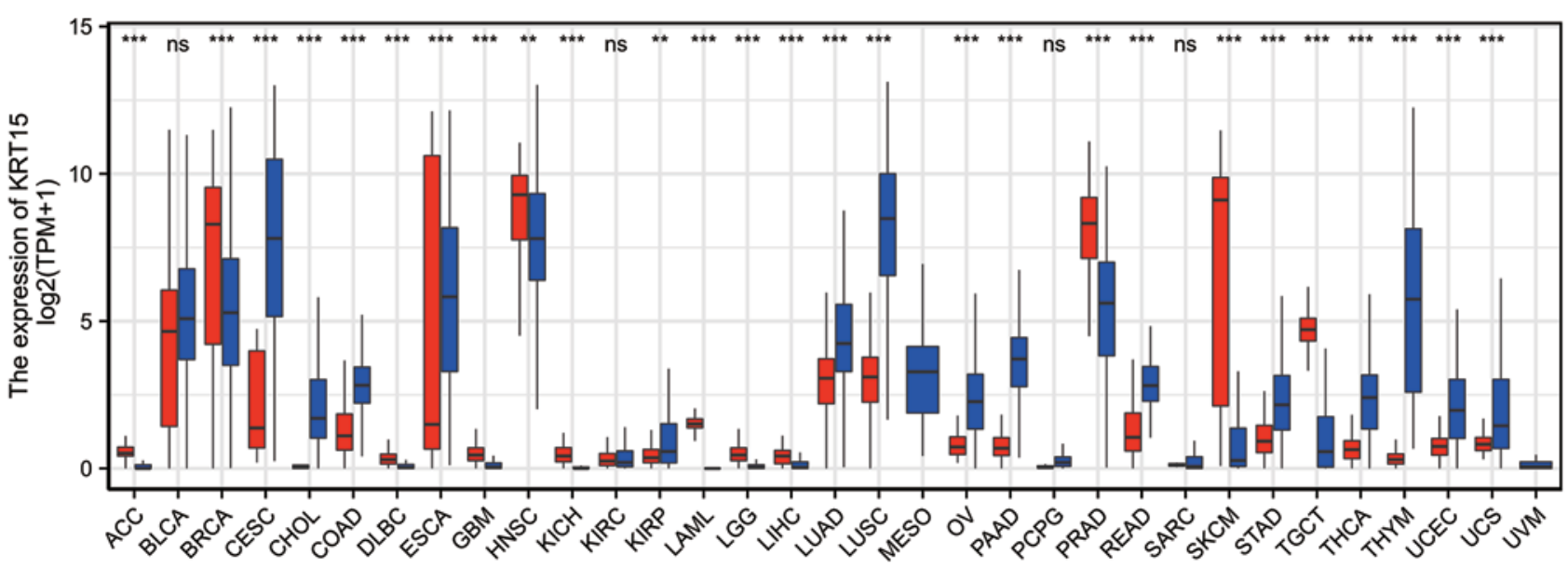

Figure 1. KRT15 expression in normal and BRCA tissues. (A) Comparison of KRT15 expression [log2(TPM+1)] between tumor and normal samples and (B) between tumor and paracancerous samples was analyzed. (C) ROC curve analysis of KRT15 expression. KRT15 expression exhibited good discriminative efficacy between tumor and non-tumor samples. (D) Pan-cancer analysis of the difference in KRT15 expression across 33 types of cancer between tumor and normal samples. ${ }^{* * *} \mathrm{P}<0.001 ; \mathrm{ns},{ }^{* *} \mathrm{P} \geq 0.05$. The abscissa presents the FPR and the ordinate the TPR. ACC, adrenocortical carcinoma; BLCA, bladder urothelial carcinoma; BRCA, breast invasive carcinoma; CESC, cervical squamous cell carcinoma and endocervical adenocarcinoma; CHOL, cholangiocarcinoma; COAD, colon adenocarcinoma; DLBC, lymphoid neoplasm diffuse large B-cell lymphoma; ESCA, esophageal carcinoma; GBM, glioblastoma multiforme; HNSC, head and neck squamous cell carcinoma; KICH, kidney chromophobe; KIRC, kidney renal clear cell carcinoma; KIRP, kidney renal papillary cell carcinoma; LAML, acute myeloid leukemia; LGG, brain lower grade glioma; LIHC, liver hepatocellular carcinoma; LUAD, lung adenocarcinoma; LUSC, lung squamous cell carcinoma; MESO, mesothelioma; OV, ovarian serous cystadenocarcinoma; PAAD, pancreatic adenocarcinoma; PCPG, pheochromocytoma and paraganglioma; PRAD, prostate adenocarcinoma; READ, rectum adenocarcinoma; SARC, sarcoma; SKCM, skin cutaneous melanoma; STAD, stomach adenocarcinoma; TGCT, testicular germ cell tumors; THCA, thyroid carcinoma; THYM, thymoma; UCEC, uterine corpus endometrial carcinoma; UCS, uterine carcinosarcoma; UVM, uveal melanoma; KRT15, keratin 15; ROC, receiver operating characteristic; AUC, area under the ROC curve; TCGA, The Cancer Genome Atlas; TPM, transcripts per million reads; FPR, false-positive rate; TPR, true-positive rate; GTEx, genotype-tissue expression.

between KRT15 expression and immune-cell infiltration levels, as calculated using ssGSEA. As presented in Fig. 4A, natural killer (NK) cells, B cells and mast cells were significantly positively correlated with KRT15 expression. Other immune cell subsets, such as eosinophils, T-helper (Th) cells and $\mathrm{T}$ regulatory (Treg) cells were significantly negatively correlated with KRT15. In addition, the Wilcoxon rank-sum test was performed to compare the immune infiltration score between the high and low KRT15 expression groups. The results presented in Fig. 4B-D respectively indicate that the 
A

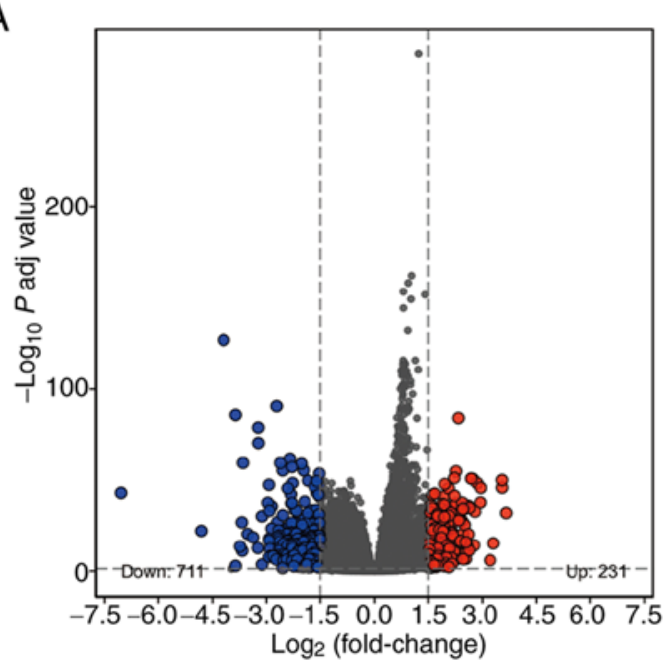

C

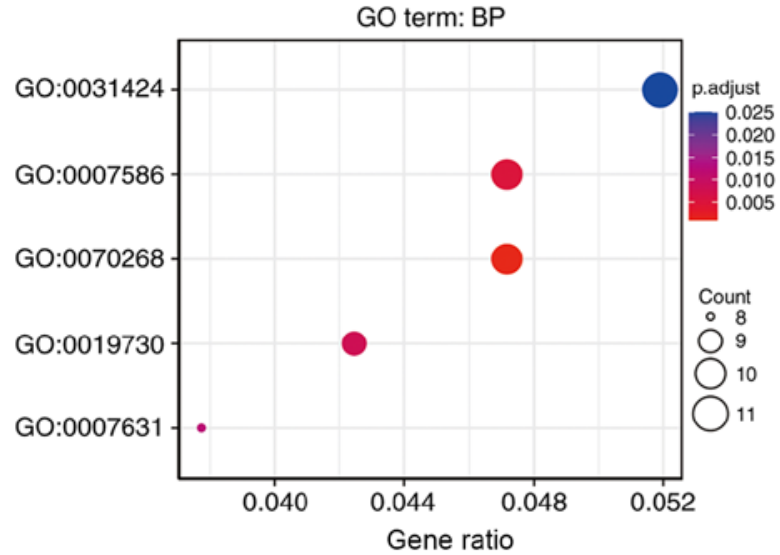

E

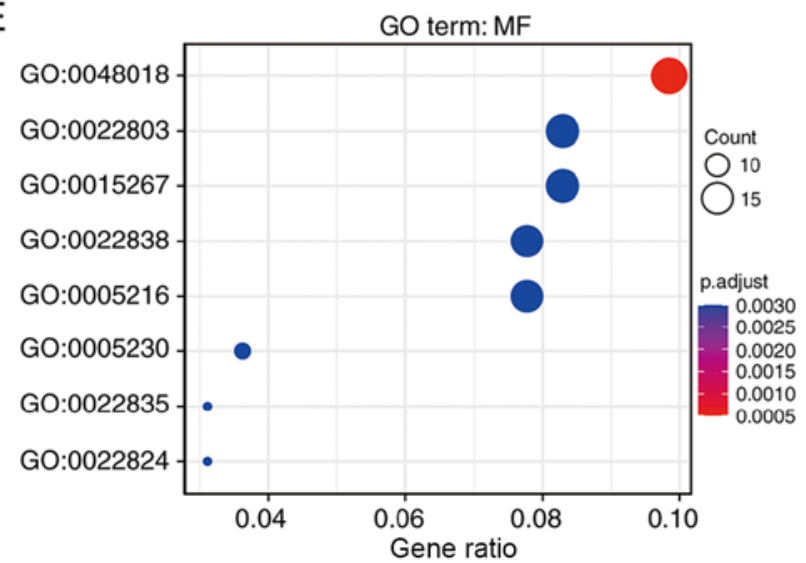

B

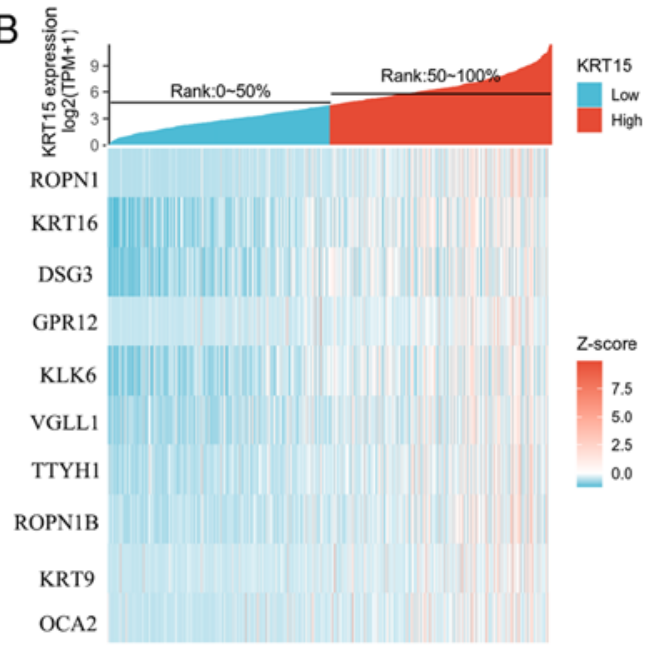

D

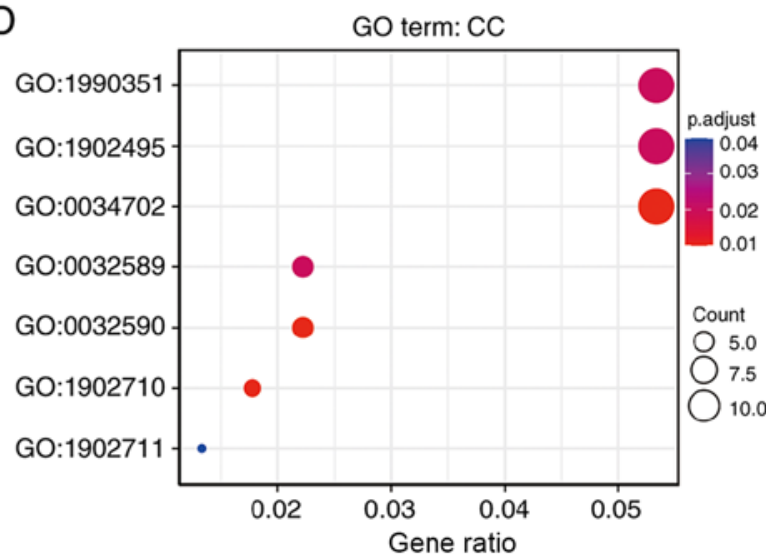

$\mathrm{F}$

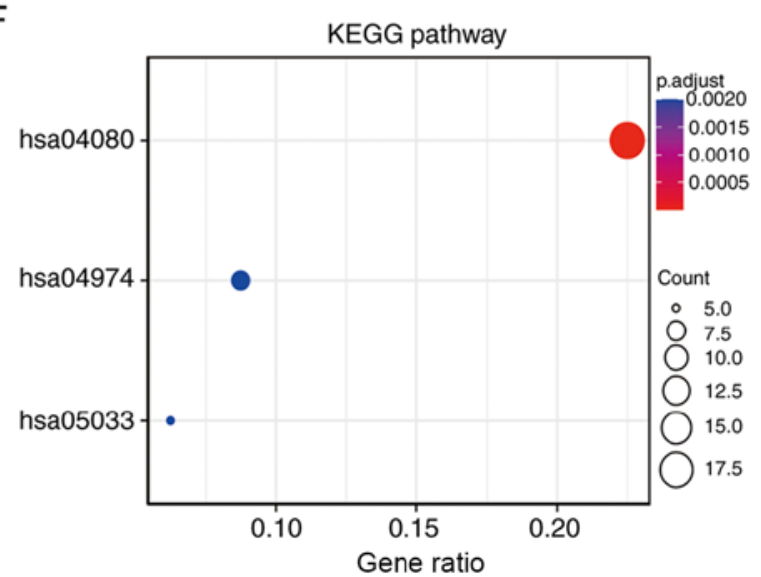

Figure 2. Functional enrichment analysis for KRT15-associated genes in BRCA. (A) Volcano plot depicting the differentially expressed genes (llog fold changel $>1.5 ; \mathrm{P}<0.05$ ) between the high and low gene expression groups. Blue represents downregulation and red upregulation. (B) Heatmap of the top differentially expressed genes in the BRCA dataset. The x-axis represents the samples and the y-axis represents the DEGs. (C-F) Bubble charts presenting significantly enriched terms of the DEGs in the GO categories (C) BP, (D) CC and (E) MF and (F) KEGG pathways. Enrichment analysis was performed based on a P adj $<0.05$. BRCA, breast invasive carcinoma; KRT15, keratin 15; FC, fold change; P adj, adjusted P-value; DEG, differentially expressed gene; TPM, transcripts per million reads; ROPN1, ropporin-1; KRT16, keratin16; DSG3, desmoglein-3; GPR12, G-protein coupled receptor 12; KLK6, kallikrein-6; VGLL1, vestigial-like protein 1; TTYH1, tweety homolog 1; KRT9, keratin 9; OCA2, oculocutaneous albinism II; GO, Gene Ontology; KEGG, Kyoto Encyclopedia of Genes and Genomes; BP, biological process; CC, cellular component; MF, molecular function.

levels of B-cell $(\mathrm{P}<0.001)$, NK-cell $(\mathrm{P}<0.001)$ and mast-cell $(\mathrm{P}<0.001)$ infiltration were significantly higher in the high KRT15 expression group compared with those in the low KRT15 expression group. Conversely, as presented in
Fig. 4E-G, respectively, the low KRT15 expression group had significantly higher levels of Th2-cell $(\mathrm{P}<0.001)$, eosinophil $(\mathrm{P}<0.001)$ and $\mathrm{Th}$ cell $(\mathrm{P}=0.003)$ infiltration compared with those in the high KRT15 expression group. 

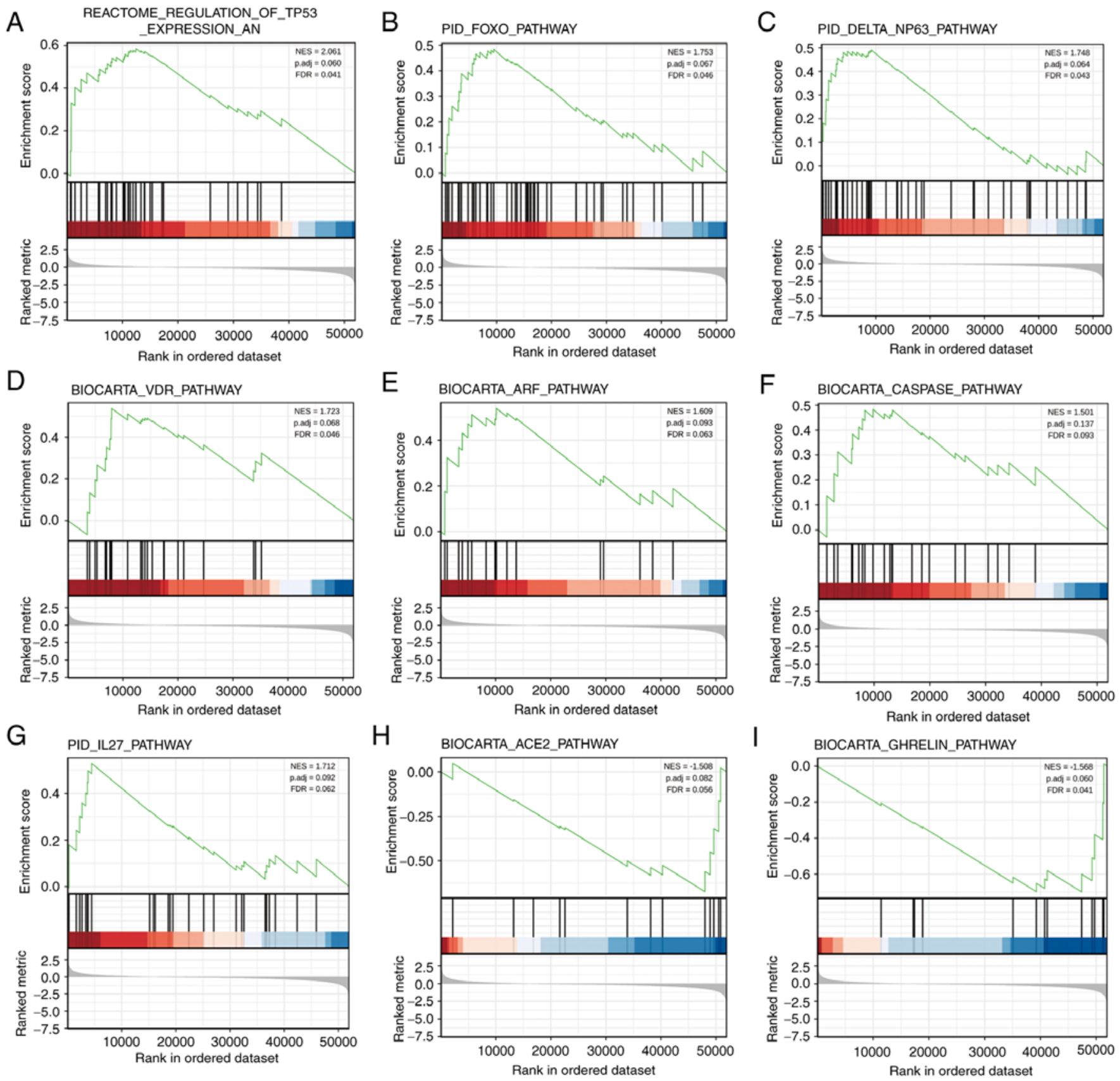

Figure 3. Enrichment map of GSEA for KRT15-related genes in BRCA. GSEA results showed datasets (A) 'REACTOME_REGULATION_OF_TP53 EXPRESSION_AND_DEGRADATION', (B) PID_FOXO_PATHWAY, (C) 'PID_DELTA_NP63_PATHWAY', (D) BIOCARTA_VDR_PATHWAY, (E) BIOCARTA_ARF_PATHWAY, (F) BIOCARTA_CASPASE_PATHWAY and (G) PID_IL27_PATHWAY were significantly enriched in the KRT15 high group, indicating a potential role of KRT15 in BRCA. Datasets (H) 'BIOCARTA_ACE2_PATHWAY' and (I) BIOCARTA_GHRELIN_PATHWAY were significantly enriched in the KRT15 low group. NES, normalized enrichment score; FDR, false discovery rate; P adj, adjusted P-value; BRCA, breast invasive carcinoma; KRT15, keratin 15; FOXO, forkhead box O; VDR, vitamin D receptor; ARF, alternative reading frame; ACE2, angiotensin converting enzyme 2.

Association between KRT15 expression and clinical characteristics. The baseline patient characteristics assessed included age, ethnicity, TNM stage, PR/ER/HER2 status, PAM50 subtype, histological type, anatomic neoplasm subdivisions, TP53 status and phosphotidylinositol4,5-bisphosphate 3-kinase catalytic subunit alpha (PIK3CA) status. The Wilcoxon rank-sum test indicated that KRT15 was significantly associated with the $\mathrm{PR}$ status $(\mathrm{P}=0.001)$, ER status $(\mathrm{P}<0.001)$, HER2 status $(\mathrm{P}<0.001)$, histological type $(\mathrm{P}<0.001)$ and $\mathrm{N}$ stage $(\mathrm{P}=0.026$; Fig. 5A-E). The Kruskal-Wallis rank-sum test also suggested that ethnicity $(\mathrm{P}=0.013)$, PAM50 subtype $(\mathrm{P}<0.001)$ and $\mathrm{T}$ stage $(\mathrm{P}=0.021)$ were significantly associated with KRT15 expression (Fig. 5F-H). The $\chi^{2}$ test indicated there was a significant difference between the low and high KRT15 expression groups in terms of PR status $(\mathrm{P}=0.004)$, ER status $(\mathrm{P}<0.001)$, HER2 status $(\mathrm{P}=0.005)$, PAM50 $(\mathrm{P}<0.001)$ and histological type $(\mathrm{P}=0.002)$. However, there was no significant difference between the low and high KRT15 expression groups in terms of $\mathrm{N}$ stage (N1, N2 and $\mathrm{N} 3$ vs. N0) and T stage (T3 and T4 vs. T1 and T2) (Table I). Similar results were observed using univariate logistic regression analysis (Table II). These results suggested that although 

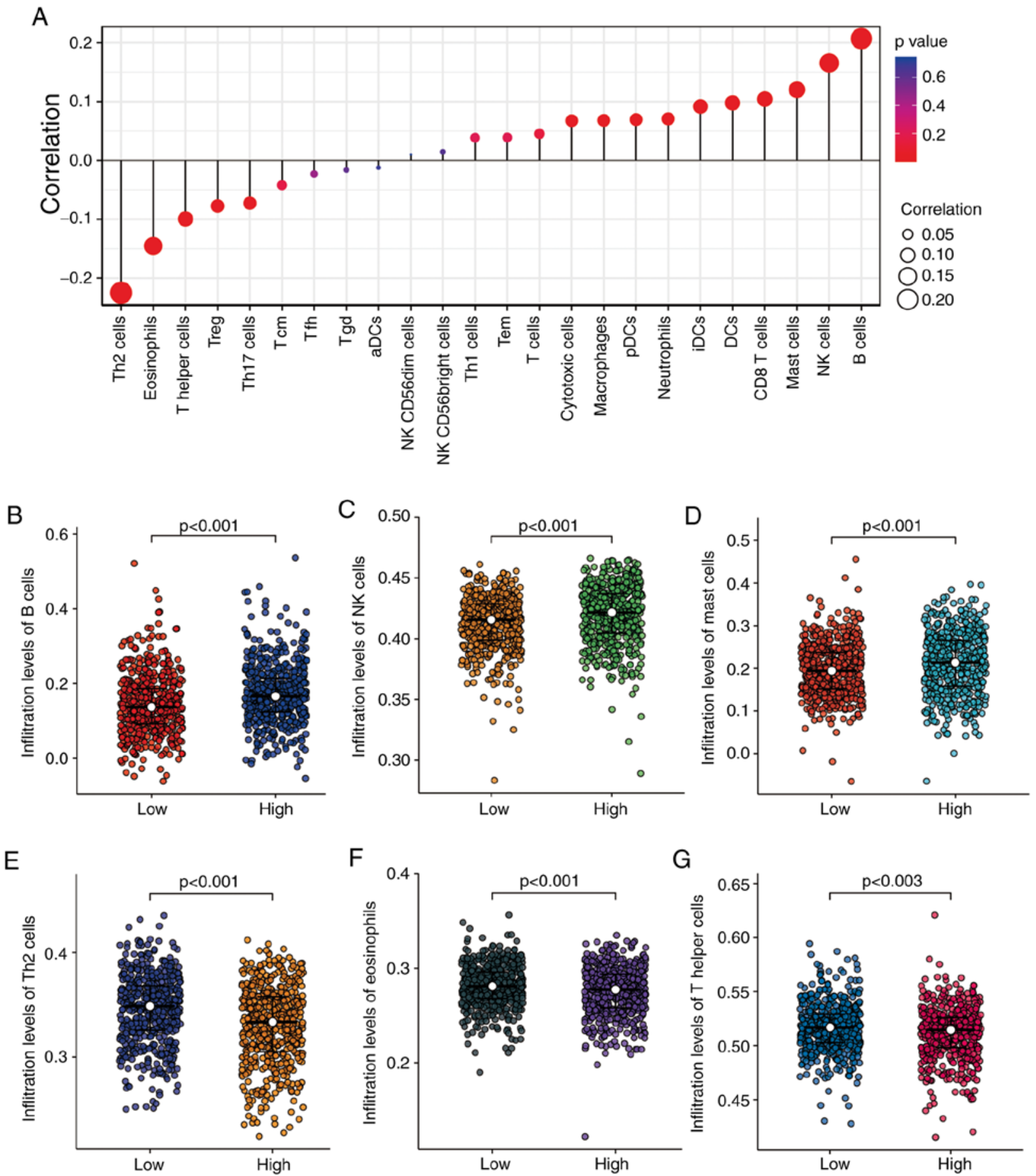

Figure 4. Correlation analyses between KRT15 expression and immune-cell infiltration levels determined by ssGSEA in BRCA. (A) Correlation between the relative abundance of 24 immune cell types and gene expression levels. The dot size represents the correlation coefficients and the color represents the P-value. (B-G) Comparison of infiltration levels of different immune cell types between high and low KRT15 expression groups. The results are presented in scatter plots: (B) B cells, (C) NK cells, (D) mast cells, (E) Th2 cells, (F) eosinophils and (G) Th cells. BRCA, breast invasive carcinoma; KRT15, keratin 15; ssGSEA, single-sample gene set enrichment analysis; NK, natural killer; Th2, type 2 T-helper; Treg, T-regulatory cells; Tcm, central memory T cell; Tfh, follicular helper T cell; Tgd, T $\gamma \delta$ cell; DCs, dendritic cells; aDCs, activated dendritic cells; pDCs, plasmacytoid dendritic cells; iDCs, immature dendritic cells; Tem, $\mathrm{T}$ effector memory.

the expression levels of KRT15 may not serve as an effective predictive index for determining $\mathrm{N}$ and $\mathrm{T}$ stage, it may serve as an index for the PR/ER/HER2 status and histological type in patients with BRCA.

Low KRT15 expression is associated with adverse outcomes in patients with BRCA. As presented in Fig. 6, KM survival analysis was used to determine the prognostic role of KRT15 in patients with BRCA. The high KRT15 expression group had significantly better OS $(\mathrm{P}=0.001$; Fig. $6 \mathrm{~A})$ and $\mathrm{DSS}(\mathrm{P}=0.005$; Fig. 6B) than the low expression group. Subsequently, the factors associated with BRCA prognosis were analyzed using a univariate Cox regression model. High KRT15 expression was significantly positively associated with favorable OS 

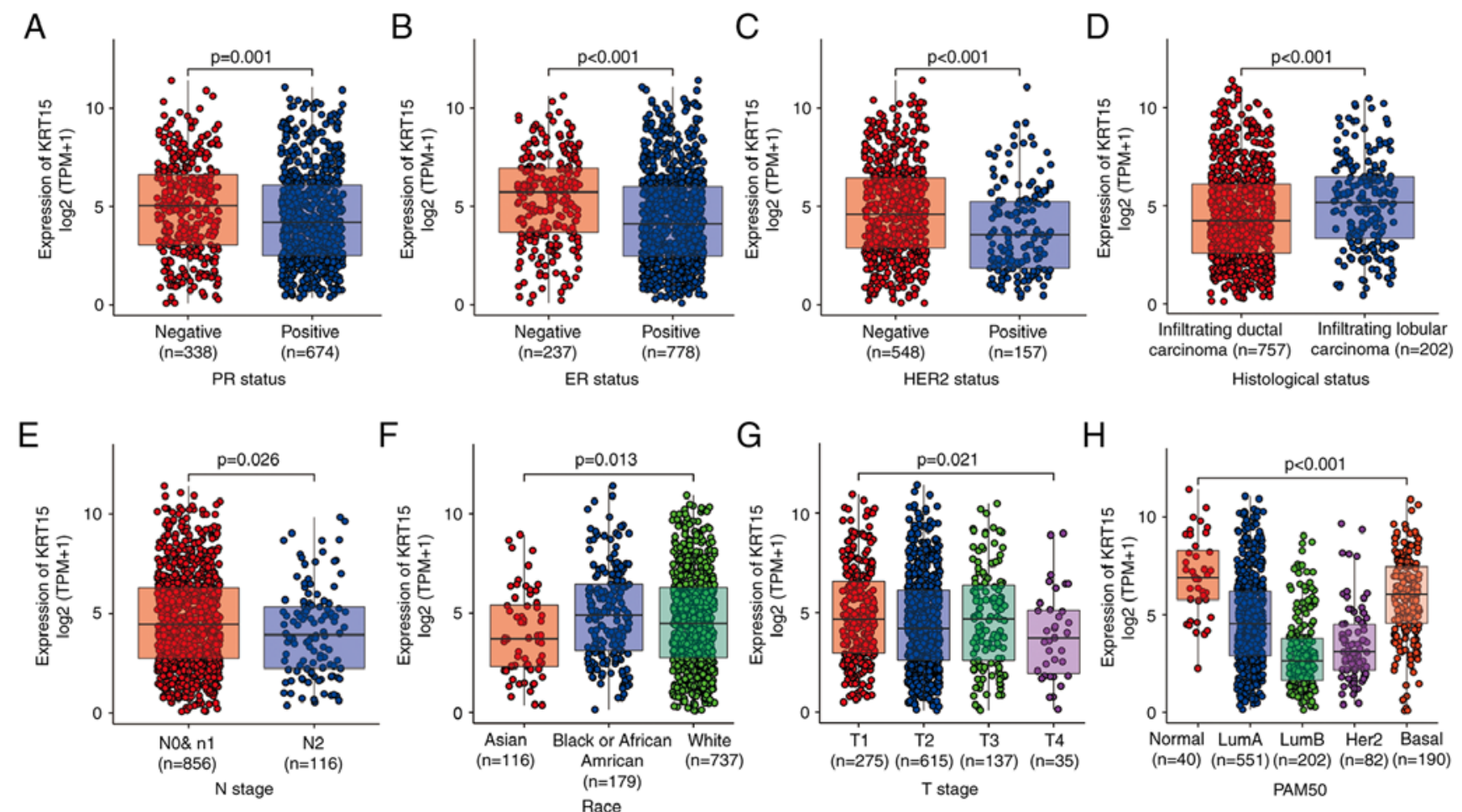

Figure 5. Association between KRT15 expression and clinicopathological features. (A) PR status, (B) ER status, (C) HER2 status, (D) histological type, (E) N stage, (F) race, (G) T stage and (H) PAM50 subtypes. KRT15, keratin 15; TPM, transcripts per million reads; PR, progesterone receptor; ER, estrogen receptor; HER2, human epidermal growth factor receptor 2; PAM50, prediction analysis of microarray 50.

Table II. Univariate logistic regression analysis of gene expression and clinicopathologic characteristics.

\begin{tabular}{lrrr}
\hline Characteristics & $\mathrm{N}$ & Odds ratio (95\% CI) & P-value \\
\hline T stage, T3/T4 vs. T1/T2 & 1,062 & $0.97(0.70-1.35)$ & 0.868 \\
N stage, N1/N2/N3 vs. N0 & 1,046 & $0.93(0.73-1.18)$ & 0.535 \\
M stage, M1 vs. M0 & 909 & $0.65(0.25-1.58)$ & 0.346 \\
Pathologic stage, III/IV vs. I/II & 1,042 & $0.86(0.65-1.14)$ & 0.297 \\
PR status, positive vs. negative & 1,012 & $0.67(0.52-0.87)$ & 0.003 \\
ER status, positive vs. negative & 1,015 & $0.43(0.31-0.58)$ & $<0.001$ \\
HER2 status, positive vs. negative & 705 & $0.58(0.41-0.84)$ & 0.004 \\
Histological type, infiltrating lobular & 959 & $1.68(1.23-2.31)$ & 0.001 \\
carcinoma vs. infiltrating ductal carcinoma & & $1.06(0.82-1.39)$ & 0.644 \\
TP53 status, Mut vs. WT & 956 & $1.11(0.85-1.46)$ & 0.441 \\
PIK3CA status, Mut vs. WT & 956 &
\end{tabular}

CI, confidence interval; PR, progesterone receptor; ER, estrogen receptor; HER2, human epidermal growth factor receptor 2; WT, wild-type; Mut, mutated; TP53, tumor protein 53; PIK3CA, phosphotidylinositol-4,5-bisphosphate 3-kinase catalytic subunit $\alpha$.

$(\mathrm{P}=0.001, \mathrm{HR}=0.581,95 \% \mathrm{CI}=0.417-0.89)$. In addition, to identify independent factors affecting OS, a multivariate Cox regression model was utilized, incorporating the TNM stage, pathological stage, ER/HER2 status and age. Patients with high KRT15 expression had significantly better OS $(\mathrm{P}<0.039$, $\mathrm{HR}=0.590,95 \% \mathrm{CI}=0.358-0.974)$ compared to patients with low KRT15 expression. The above results demonstrated that KRT15 is an independent prognostic factor for OS (Table III).

Furthermore, a nomogram prognostic model was constructed to predict OS and DSS. Based on multivariate analysis, independent prognostic factors including KRT15, $\mathrm{T}$ stage, $\mathrm{M}$ stage, ER status and age were analyzed in combination. The discriminatory efficiency of the prognostic factors was evaluated using the $\mathrm{C}$-index. The $\mathrm{C}$-index of the nomogram for OS (Fig. S2A) and DSS (Fig. S2C) was 0.717 (95\% CI=0.692-0.742) and 0.775 (95\% CI=0.745-0.805), respectively. A calibration plot demonstrated the predicted values and real values of the model. The corrected line is close to the reference line, indicating a satisfactory match between the predictions and observations. In summary, these results 
A
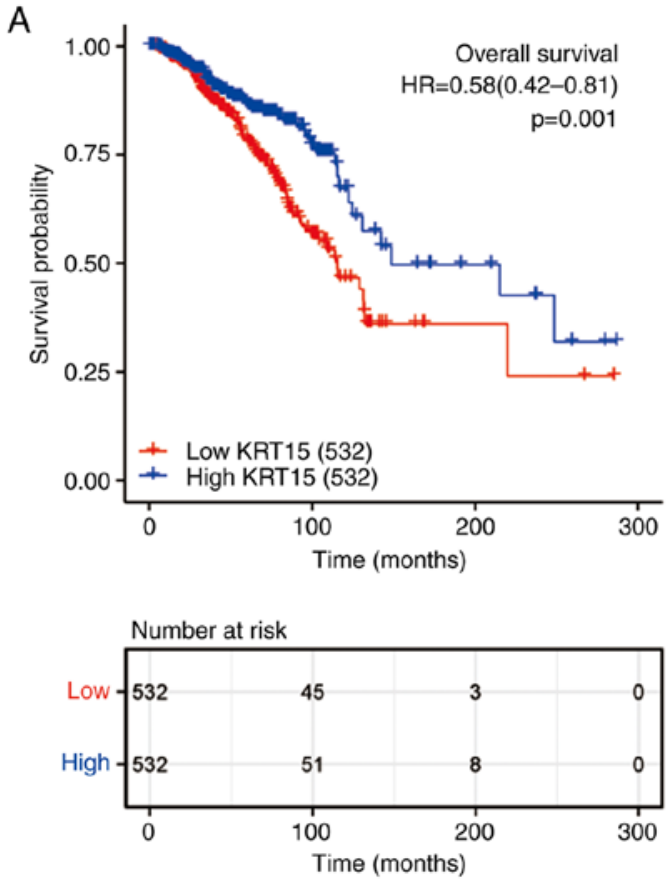

B
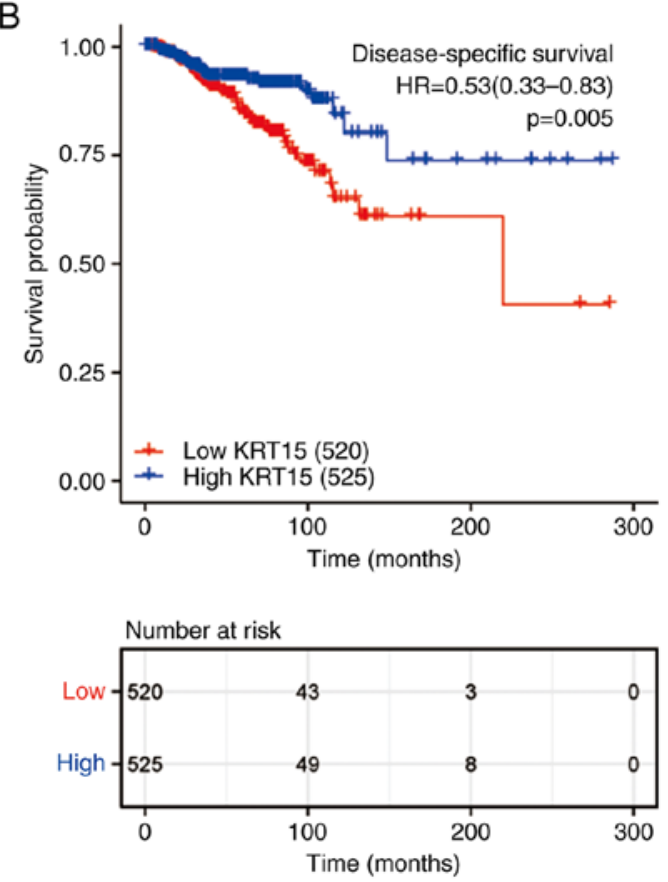

Figure 6. Effect of KRT15 expression on OS and DSS based on data obtained from The Cancer Genome Atlas. (A) The 300-month OS rate of patients with BRCA and high KRT15 expression was significantly higher compared with that in the low KRT15 expression group (P=0.001; HR=0.58; $95 \% \mathrm{CI}$, 0.42-0.81). (B) The 300-month DSS rate in patients with BRCA and high KRT15 expression was significantly higher compared with that of patients with low KRT15 expression $(\mathrm{P}=0.005$; HR=0.53; 95\% CI, 0.33-0.83). DSS, disease-specific survival; OS, overall survival; BRCA, breast invasive carcinoma; KRT15, keratin 15; HR, hazard ratio; CI, confidence interval.

Table III. Association between clinicopathological characteristics and overall survival determined by univariate and multivariate Cox regression analysis.

\begin{tabular}{|c|c|c|c|c|c|}
\hline \multirow[b]{2}{*}{ Characteristics } & \multirow[b]{2}{*}{$\mathrm{N}$} & \multicolumn{2}{|l|}{ Univariate } & \multicolumn{2}{|c|}{ Multivariate } \\
\hline & & HR $(95 \%$ CI $)$ & P-value & $\mathrm{HR}(95 \% \mathrm{CI})$ & P-value \\
\hline T stage, $\mathrm{T} 1 / \mathrm{T} 2$ vs. T3/T4 & 1,061 & $0.598(0.412-0.868)$ & 0.007 & $0.413(0.212-0.806)$ & 0.01 \\
\hline $\mathrm{N}$ stage, $\mathrm{N} 1 / \mathrm{N} 2 / \mathrm{N} 3$ vs. N0 & 1,045 & $2.145(1.497-3.073)$ & $<0.001$ & $1.342(0.719-2.505)$ & 0.355 \\
\hline M stage, M1 vs. M0 & 909 & $4.327(2.508-7.465)$ & $<0.001$ & $2.677(1.045-6.858)$ & 0.04 \\
\hline Pathologic stage, III/IV vs. I/II & 1,041 & $2.519(1.787-3.549)$ & $<0.001$ & $2.041(0.968-4.302)$ & 0.061 \\
\hline PR status, positive vs. negative & 1,011 & $0.762(0.541-1.074)$ & 0.120 & & \\
\hline ER status, positive vs. negative & 1,014 & $0.704(0.487-1.017)$ & 0.062 & $0.402(0.240-0.674)$ & $<0.001$ \\
\hline HER2 status, positive vs. negative & 705 & $1.611(0.981-2.644)$ & 0.059 & $0.910(0.509-1.628)$ & 0.751 \\
\hline Age,$>60$ vs. $\leq 60$ years & 1,064 & $2.036(1.468-2.822)$ & $<0.001$ & $3.267(1.974-5.407)$ & $<0.001$ \\
\hline $\begin{array}{l}\text { Ethnicity, white vs. Asian/black or } \\
\text { African American }\end{array}$ & 975 & $0.880(0.593-1.306)$ & 0.526 & & \\
\hline $\begin{array}{l}\text { Histological type, infiltrating ductal carcinoma } \\
\text { vs. infiltrating lobular carcinoma }\end{array}$ & 959 & $1.162(0.738-1.830)$ & 0.516 & & \\
\hline Anatomic neoplasm subdivisions, right vs. left & 1,064 & $0.776(0.559-1.077)$ & 0.13 & & \\
\hline TP53 status, Mut vs. WT & 955 & $1.218(0.858-1.730)$ & 0.269 & & \\
\hline PIK3CA status, Mut vs. WT & 955 & $1.015(0.696-1.479)$ & 0.938 & & \\
\hline KRT15, high vs. low & 1,064 & $0.581(0.417-0.809)$ & 0.001 & $0.590(0.358-0.974)$ & 0.039 \\
\hline
\end{tabular}

PR, progesterone receptor; ER, estrogen receptor; HER2, human epidermal growth factor receptor 2; WT, wild-type; Mut, mutated; TP53, tumor protein 53; PIK3CA, phosphotidylinositol-4,5-bisphosphate 3-kinase catalytic subunit $\alpha$; KRT15, keratin 15.

suggested that the nomogram was a suitable discriminatory model for predicting OS (Fig. S2B) and DSS (Fig. S2D) in BRCA.
KRT15 expression affects BRCA prognosis in patients with different demographics and in pathological subgroups. To further delineate the potential relevance and possible 
A

\begin{tabular}{|c|c|c|c|c|}
\hline Characteristics & $\mathrm{N}(\%)$ & $\mathrm{HR}(95 \% \mathrm{Cl})$ & & $\mathrm{P}$ value \\
\hline \multicolumn{5}{|l|}{ T stage } \\
\hline T1 & $274(26)$ & $0.959(0.480-1.915)$ & $\longmapsto$ & 0.905 \\
\hline T2 & $615(58)$ & $0.620(0.390-0.985)$ & • & 0.043 \\
\hline T3\&T4 & $172(16)$ & $0.345(0.173-0.691)$ & $\longmapsto$ & 0.003 \\
\hline \multicolumn{5}{|l|}{$\mathrm{N}$ stage } \\
\hline NO & 507 (49) & $0.565(0.309-1.035)$ & $\longmapsto$ & 0.064 \\
\hline N1\&N2\&N3 & $538(51)$ & $0.594(0.390-0.904)$ & $\mapsto \bullet$ & 0.015 \\
\hline \multicolumn{5}{|l|}{ M stage } \\
\hline MO & $889(98)$ & $0.599(0.416-0.864)$ & & 0.006 \\
\hline
\end{tabular}
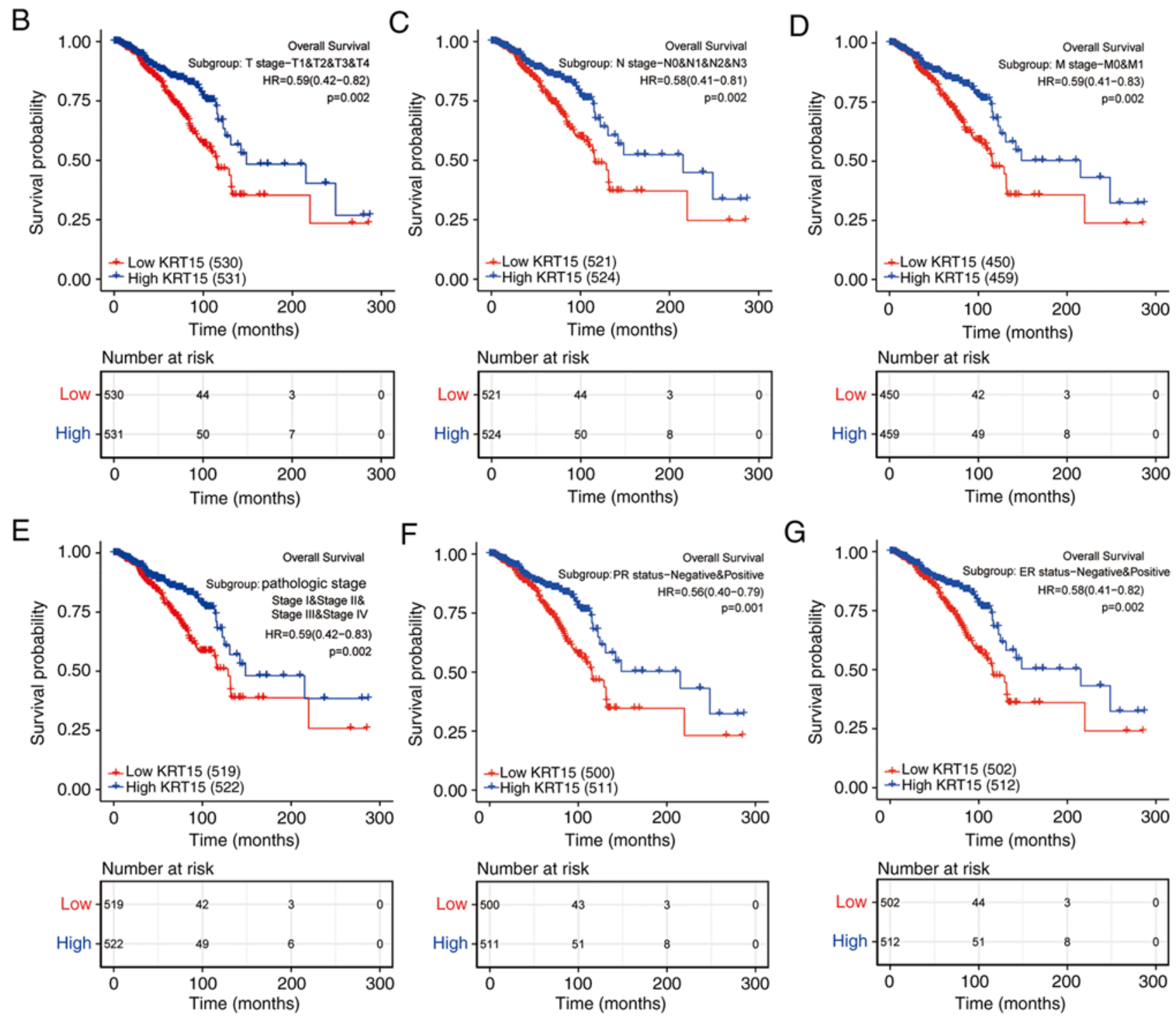

Figure 7. Forest and KM plots for OS based on KRT15 expression in the different clinicopathological subgroups. (A) Forest plot of univariate Cox analysis on OS for different clinicopathological subgroups based on KRT15 expression in BRCA. (B-G) KM survival plots based on KRT15 expression in clinicopathological subgroups of patients with BRCA. (B) T1-4, (C) N0-3, (D) M0 and M1, (E) pathologic stages I-IV, (F) PR-negative and PR-positive and (G) ER-negative and ER-positive. HRs are presented with the 95\% CI. KM, Kaplan-Meier; KRT15, keratin 15; OS, overall survival; BRCA, breast invasive carcinoma; PR, progesterone receptor; ER, estrogen receptor; HR, hazard ratio; $\mathrm{CI}$, confidence interval.

mechanisms of KRT15 expression in BRCA, the association of the KRT15 expression levels with the outcomes of different clinicopathological subgroups was assessed using Cox regression analysis. High KRT15 expression was associated with better OS compared with low KRT15 expression, in the following stages: $\mathrm{T} 2(\mathrm{HR}=0.620,95 \% \mathrm{CI}=0.390-0.985$, $\mathrm{P}=0.043), \mathrm{T} 3$ and $\mathrm{T} 4(\mathrm{HR}=0.345,95 \% \mathrm{CI}=0.173-0.691$, $\mathrm{P}=0.003), \mathrm{N} 1-3(\mathrm{HR}=0.594,95 \% \mathrm{CI}=0.390-0.904, \mathrm{P}=0.015)$ and M0 (HR=0.599, 95\% CI=0.416-0.864, $\mathrm{P}=0.006$; Fig. 7A). Among all comparisons performed, high KRT15 expression 
A
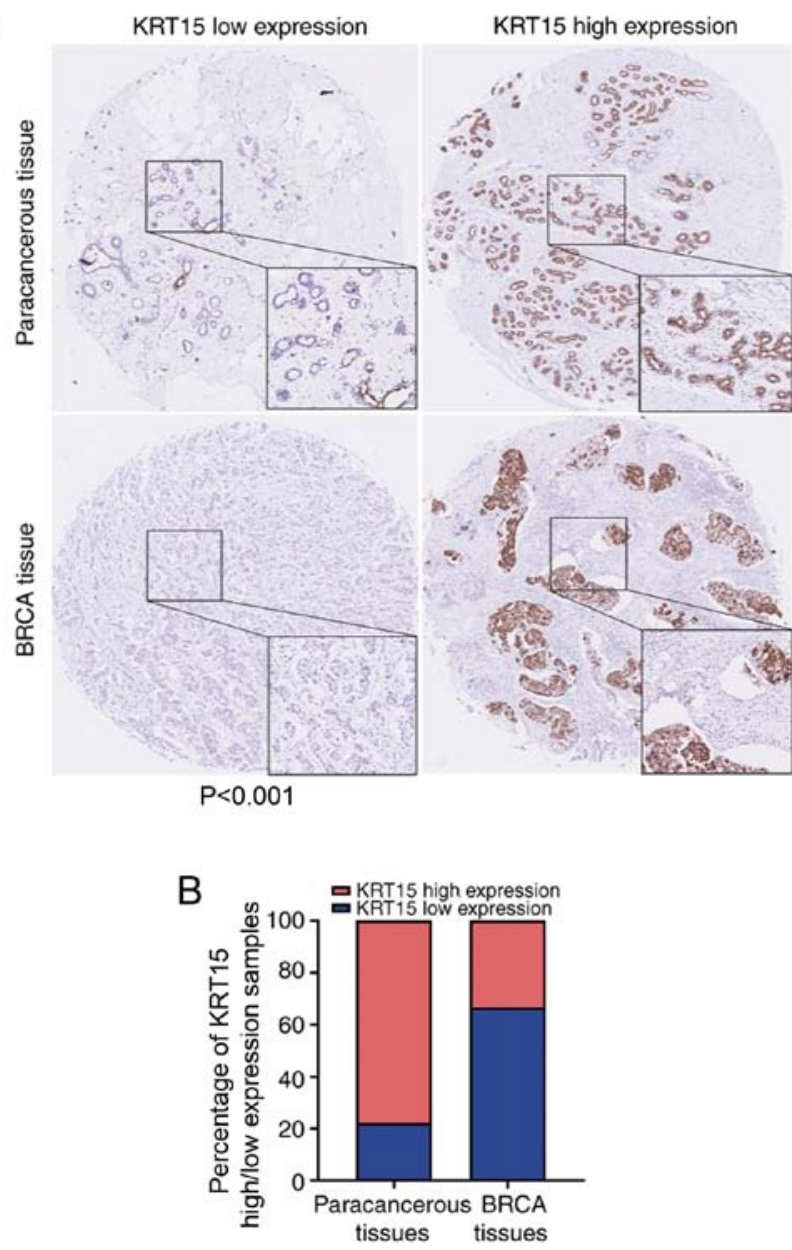

Figure 8. Representative images of KRT15 expression in BRCA tissues and paracancerous tissues. (A) KRT15 low (top left image) or high expression (top right image) in paracancerous tissues. KRT15 low (bottom left image) or high expression (bottom right image) in BRCA tissues. Original magnification, x200 and x400 (magnified windows). (B) Histogram providing the percentage of KRT15 high/low expression samples in the BRCA tissue microarray. BRCA, breast invasive carcinoma; KRT15, keratin 15 .

had the lowest HR value for OS in patients at stage T3 and T4. Similar to the above analysis on OS, high KRT15 expression was associated with improved DSS (Fig. S3A).

The prognostic value of KRT15 for OS in various subgroups of BRCA was further determined using KM analysis. Low KRT15 expression was highly associated with unfavorable prognosis in the following stages/classifications: T1-4 ( $\mathrm{P}=0.002$; Fig. 7B), N0-3 ( $\mathrm{P}=0.002$; Fig. 7C), $\mathrm{M} 0$ and M1 ( $\mathrm{P}=0.002$; Fig. 7D), pathological stage I-IV ( $\mathrm{P}=0.002$; Fig. 7E), PR-negative and PR-positive $(\mathrm{P}=0.001$; Fig. 7F) and ER-negative and ER-positive $(\mathrm{P}=0.002$; Fig. 7G). As presented in Fig. S3B-I, low KRT15 expression was associated with the following characteristics: Asian and black or African American and white ethnicity ( $\mathrm{P}=0.003)$, age $\leq 60$ and $>60$ years $(\mathrm{P}=0.001)$, histological types of infiltrating ductal carcinoma and infiltrating lobular carcinoma $(\mathrm{P}=0.014)$, HER2-negative and HER2-positive ( $\mathrm{P}=0.028)$, the PAM50 normal and luminal (Lum)A and LumB and HER2 and basal subtypes $(\mathrm{P}=0.001)$, anatomic neoplasm subdivisions left and right $(\mathrm{P}=0.001)$, TP53 status wild-type (WT) and mutated (Mut) $(\mathrm{P}<0.001)$ and PIK3CA status WT and Mut $(\mathrm{P}<0.001)$.
These results indicated that KRT15 expression may affect the prognosis of patients with BRCA in different demographic and pathological subgroups.

KRT15 expression in tissue microarrays is consistent with that based on data obtained from TCGA. To further evaluate the clinical significance of KRT15 expression, IHC staining for KRT15 was performed in a tissue microarray containing BRCA and paracancerous tissues from 90 cases. All patients were female with an average age $54.8 \pm 12.5$ years. Amongst the BRCA tissue samples, 66.7\% (30/45) did not exhibit any marked KRT15 expression. By contrast, high KRT15 expression was observed in $77.8 \%$ (35/45) of the paracancerous tissues. Compared with that among the BRCA tissue samples, the ratio of tissues with high KRT15 expression was significantly higher among the paracancerous tissue samples $(\mathrm{P}<0.001)$. The results and representative images are provided in Fig. 8.

\section{Discussion}

KRT, an intermediate filament protein, serves an important role in maintaining the structural integrity of epithelial cells. KRT has at least 20 subtypes, termed KRT1-20, and the expression patterns of each KRT filament are frequently distinctive (22). KRT15 belongs to the complex epithelial KRT family of proteins and is involved in epithelial lineage differentiation, tissue regeneration (23) and wound repair $(24,25)$. It has also been reported that KRT15 has different expression characteristics across different types of cancer. KRT15-expressing cells in the hair follicle bulge promote the development of squamous papilloma (26) and basal cell carcinomas (27). KRT15 expression is increased in squamous cell lung carcinoma (28), colorectal cancer (11) and a subset of urothelial cell carcinomas (29). In addition, the expression of KRT15 is positively associated with the OS time of patients with gastric cancer (30) and greater positivity for KRT15 generally favors benign lesions (31). However, the expression levels and prognostic significance of KRT15 in BRCA have remained largely elusive. Therefore, the aim of the present study was to assess the clinical significance of KRT15 in BRCA. Through a series of analyses, it was determined whether KRT15 may be used as a candidate prognostic marker for BRCA.

Cornification (also known as keratinization) is the final form of epithelial cell differentiation and is regarded as a special type of squamous epithelial cell apoptosis in the upper layer of the epithelial tissue (32-34). Cornification serves an important role in maintaining normal epithelial cell differentiation/maturation. Therefore, abnormal or absent cornification promotes malignant cell transformation $(35,36)$. In the present study, GO enrichment analysis suggested that KRT15-associated DEGs were significantly enriched in cornification (keratinization). This result is consistent with the Reactome pathway database (reactome.org) regarding KRT15-related pathways (Table SII). Thus, KRT15 was indicated to be involved in cornification to maintain epidermal homeostasis in BRCA.

Furthermore, analysis of data obtained from TCGA for GSEA indicated a significantly positive association between high expression of KRT15 and tumor-suppressive pathways, 
such as regulation of TP53 expression and degradation, the FOXO pathway, the $\triangle$ NP63 pathway, the VDR pathway, the ARF pathway, the caspase pathway and the IL27 pathway. TP53 has been extensively demonstrated to function as a tumor suppressor gene and loss of TP53 function is a characteristic feature of numerous types of cancer $(37,38)$. FOXO is a member of the subfamily of the forkhead transcription factor family with important functions in cell fate determination and this subfamily also serves a critical role as tumor suppressors in several types of cancer (39). $\triangle \mathrm{NP} 63$ is a P53 family member and regulates the expression of various genes crucial for inhibiting tumorigenesis (40-42). Numerous studies have demonstrated that expression of the VDR pathway is associated with favorable prognosis of patients with cancer, particularly colorectal cancer $(43,44)$. The ARF pathway may attenuate cell DNA damage and this characteristic is known to be responsible for its anti-tumor response (45). The caspase pathway is essential for the normal development and function of multicellular organisms, as abnormal cell death control may lead to a variety of diseases, including cancer (46). IL-27 enhances the functions of $\mathrm{Th} 1$ and $\mathrm{CD} 8^{+} \mathrm{T}$ cells; promotes the development of follicular helper $\mathrm{T}$ cell and $\mathrm{T}$ regulatory 1 , which could activate $\mathrm{B}$ cells to proliferate and secrete antibodies; and inhibits the functions of Th2, Treg and Th17 cells. Therefore, IL-27 has a tumor suppressor role in cancer (47). This illustrates the functional interdependence between upregulated KRT15 expression and the normal tumor suppressor function of these pathways in BRCA. Of note, the ACE2 pathway and the ghrelin pathway were significantly enriched in the low KRT15 expression group according to the GSEA analysis. A pan-cancer analysis of TCGA datasets demonstrated that ACE2 was expressed at a significantly higher level in BRCA compared to normal samples (48). Studies have reported that ghrelin may promote the expression of oncogene and also induce tumor resistance (49,50). GO enrichment analysis suggested that KRT15 is associated with the receptor-ligand complex and transmembrane transporter complex. This suggests that KRT15 may influence the expression of ACE2 and ghrelin by interacting with the cell membrane complex.

Of note, KRT15 significantly influences immune-cell infiltration in BRCA. In the present study, ssGSEA and Spearman's rank correlation analysis revealed significant connections between the KRT15 expression levels and immune-cell infiltration. In particular, KRT15 expression was positively correlated with NK cells, mast cells and B cells, and negatively correlated with eosinophils, Th cells and Treg cells. Previous studies have indicated that the absence of NK cells increases the incidence of all types of cancer (51) and may also significantly accelerate tumor growth $(52,53)$. It is known that cytotoxicity and the release of cytokines are the two major functions of NK cells and cytokine production can lead to the activation of NK cells, promoting their antitumor effects (54). IFN- $\gamma$ and TNF- $\alpha$ secreted from NK cells contribute to the suppression of tumor proliferation and angiogenesis (55). In addition, through the production of chemokines $\mathrm{C}-\mathrm{C}$ motif ligand 1 and lymphotactin $\alpha / \beta$ NK cells recruit more dendritic cells into solid tumors, which improves the prognosis of patients (56). Mast cells are also considered to be unfavorable for tumor growth due to their ability to secrete IL-4, TNF- $\alpha$ and other cytokines to elicit tumor-cell apoptosis $(57,58)$. A series of studies have indicated that B cells and B cell-derived tertiary lymphoid structures serve an important role in cancer immunity and contribute to improved immunotherapy (59-61). Thus, it may be hypothesized that KRT15 overexpression promotes efficient NK-cell and mast-cell immune infiltration and initiates an antitumor immune response.

Th cell subsets (Th1, Th2, Th17 and Treg cells) serve a critical role in cancer immunity (62). Th2 cells produce and secrete IL-4 and IL-10, which have been indicated to stimulate tumor-cell proliferation and promote tumor-cell resistance to apoptosis $(63,64)$. Treg cells are able to induce a tumor suppressive microenvironment and foster tumor growth and recurrence by suppressing tumor-reactive T cells and NK cells $(65,66)$. IL-17 derived from Th17 cells is able to activate the STAT3 pathway in tumors and mediate a myeloid-derived suppressor cell-induced, tumor-promoting microenvironment (67). In the present study, GO and KEGG enrichment analysis indicated that KRT15 is closely correlated with various cell-membrane complexes, including the receptor-ligand complex. Taken together, these results suggest that KRT15 may affect the binding of immune-cell ligands to their receptors and serve a distinct role in regulating immune-cell infiltration. Therefore, elevated KRT15 may ultimately influence the prognosis of patients with BRCA.

In the present study, it was demonstrated that the expression levels of KRT15 in BRCA tissues were significantly lower compared with those in normal tissues and may serve as a predictive index for determining the PR/ER/HER2 status and histological type in patients with BRCA. Furthermore, lower KRT15 expression was associated with poor OS or DSS. In addition, univariate and multivariate Cox regression analyses indicated that KRT15 may be used as an independent prognostic factor for patients with BRCA after adjustment for conventional clinical characteristics. Furthermore, a nomogram was constructed by combining KRT15 expression with other important clinical factors (T/M stage, ER status and age), which exhibited relatively accurate prediction efficiency for BRCA. The calibration curve had a satisfactory fit for predicting the 1-,3- and 5-year survival of patients with BRCA. Furthermore, a subgroup analysis of the association between KRT15 expression and clinicopathological factors was performed. Low KRT15 expression exhibited a significant association with the T4 stage, N2 stage, infiltrating ductal carcinoma and positive $\mathrm{PR} / \mathrm{ER} / \mathrm{HER} 2$ status. These results demonstrated that low KRT15 expression was not only positively associated with BRCA, but also positively associated with an advanced tumor stage and more aggressive BRCA subtype. To further assess the relationship between KRT15 expression and OS in patients with BRCA, KM survival analysis was performed on subgroups of patients with BRCA stratified according to KRT15 expression levels and by clinicopathological factors. The results revealed a significant association between low KRT15 expression and poor OS in all of the selected clinical subgroups. In short, these results indicated that KRT15 may be considered a strong predictor of OS within these subgroups. Patients with BRCA were able to be classified 
into low-risk and high-risk groups according to their KRT15 expression levels. Finally, to further validate the results from TCGA, IHC analysis was used to determine KRT15 protein expression in BRCA. These results also indicated that the positive KRT15 expression rate was lower in BRCA tissues compared with that in normal tissues, consistent with the results of the TCGA analysis.

Although the present study has provided a deeper understanding of the relationship between KRT15 and BRCA, there are several limitations. First, the present study was performed based on RNA sequences obtained from one database and the comprehensiveness of the data is not guaranteed; therefore, controlled and multi-center trials are required. Furthermore, the precise mechanism by which KRT15 affects the prognosis of patients with BRCA requires further study. Finally, the diagnostic role and therapeutic utility of KRT15 in BRCA remain to be determined and further exploration is required to determine the potential clinical applications of KRT15.

In conclusion, KRT15 may be an independent predictor of favorable prognosis for patients with BRCA. It has a role in the regulation of tumor suppressor pathways and anti-tumoral immune-cell infiltration in BRCA. Additional research is necessary to elucidate the underlying direct mechanisms involved in these processes. Furthermore, follow-up studies are required to comprehensively assess the association between KRT15 and clinical factors of BRCA using other large databases or more than one database. The present results suggest that KRT15 may be used as a novel biomarker to predict the tumor stage and prognosis of patients with BRCA, to improve treatment outcomes and as a novel drug target.

\section{Acknowledgements}

Not applicable.

\section{Funding}

The present study was financially supported by the funds from Science and Technology Projects of Guangdong Province (grant no. 2016A020217015), the Department of Education of Guangdong Province (grant no. 2015KCXTD023) and the Guangzhou University of Chinese Medicine (grant no. A3-0402-008), China.

\section{Availability of data and materials}

The datasets used and/or analyzed during the current study are available from the corresponding author on reasonable request.

\section{Authors' contribution}

YH and PZ designed the study. RS and HW performed a database search with data retrieval and cleaning. PZ and ZL performed data analysis and interpretation. XS contributed to the supervision of the study and was involved in generating figures and tables. PZ performed the IHC analysis of the tissue microarray and wrote the manuscript. All authors read and approved the final manuscript.

\section{Ethical approval and consent to participate}

Not applicable.

\section{Patient consent for publication}

Not applicable.

\section{Competing interest}

The authors declare that they have no competing interests.

\section{References}

1. Siegel RL, Miller KD and Jemal A: Cancer statistics, 2019. CA Cancer J Clin 69: 7-34, 2019.

2. Malvezzi M, Bertuccio P, Levi F, La Vecchia C and Negri E: European cancer mortality predictions for the year 2013. Ann Oncol 24: 792-800, 2013.

3. Polyak K: Heterogeneity in breast cancer. J Clin Invest 121: 3786-3788, 2011.

4. Klimczak M, Biecek P, Zylicz A and Zylicz M: Heat shock proteins create a signature to predict the clinical outcome in breast cancer. Sci Rep 9: 7507, 2019.

5. Azizi M, Ghourchian H, Yazdian F, Bagherifam S, Bekhradnia S and Nyström B: Anti-cancerous effect of albumin coated silver nanoparticles on MDA-MB 231 human breast cancer cell line. Sci Rep 7: 5178, 2017.

6. Wang J, Yin H, Panandikar A, Gandhi V and Sen S: Elevated cyclin A associated kinase activity promotes sensitivity of metastatic human cancer cells to DNA antimetabolite drug. Int J Oncol 47: 782-790, 2015.

7. Masica DL and Karchin R: Correlation of somatic mutation and expression identifies genes important in human glioblastoma progression and survival. Cancer Res 71: 4550-4561, 2011.

8. Liu Y, Lyle S, Yang Z and Cotsarelis G: Keratin 15 promoter targets putative epithelial stem cells in the hair follicle bulge. J Invest Dermatol 121: 963-968, 2003.

9. Nagosa S, Leesch F, Putin D, Bhattacharya S, Altshuler A, Serror L, Amitai-Lange A, Nasser W, Aberdam E, Rouleau M, et al: microRNA-184 induces a commitment switch to epidermal differentiation. Stem Cell Reports 9: 1991-2004, 2017.

10. Sanchez-Palencia A, Gomez-Morales M, Gomez-Capilla JA, Pedraza V, Boyero L, Rosell R and Fárez-Vidal ME: Gene expression profiling reveals novel biomarkers in nonsmall cell lung cancer. Int J Cancer 129: 355-364, 2011.

11. Rao X, Wang J, Song HM, Deng B and Li JG: KRT15 overexpression predicts poor prognosis in colorectal cancer. Neoplasma 67: 410-414, 2020.

12. Seykora JT and Cotsarelis G: Keratin 15-positive stem cells give rise to basal cell carcinomas in irradiated $\mathrm{Ptchl}^{+/-}$mice. Cancer Cell 19: 5-6, 2011.

13. Moon H, Zhu J, Donahue LR, Choi E and White AC: $\mathrm{Krt}^{+} / \mathrm{Krt}^{+} 5^{+}$ foregut basal progenitors give rise to cyclooxygenase-2-dependent tumours in response to gastric acid stress. Nat Commun 10: $2225,2019$.

14. Vivian J, Rao AA, Nothaft FA, Ketchum C, Armstrong J, Novak A, Pfeil J, Narkizian J, Deran AD, Musselman-Brown A, et al: Toil enables reproducible, open source, big biomedical data analyses. Nat Biotechnol 35: 314-316, 2017.

15. Robin X, Turck N, Hainard A, Tiberti N, Lisacek F, Sanchez JC and Müller M: pROC: An open-source package for R and S+ to analyze and compare ROC curves. BMC Bioinformatics 12: 77, 2011.

16. Love MI, Huber W and Anders S: Moderated estimation of fold change and dispersion for RNA-seq data with DESeq2. Genome Biol 15: 550, 2014

17. Yu G, Wang LG, Han Y and He QY: clusterProfiler: An R package for comparing biological themes among gene clusters. OMICS 16: 284-287, 2012. 
18. Subramanian A, Tamayo P, Mootha VK, Mukherjee S, Ebert BL Gillette MA, Paulovich A, Pomeroy SL, Golub TR, Lander ES and Mesirov JP: Gene set enrichment analysis: A knowledge-based approach for interpreting genome-wide expression profiles. Proc Natl Acad Sci USA 102: 15545-15550, 2005.

19. Bindea G, Mlecnik B, Tosolini M, Kirilovsky A, Waldner M, Obenauf AC, Angell H, Fredriksen T, Lafontaine L, Berger A, et al: Spatiotemporal dynamics of intratumoral immune cells reveal the immune landscape in human cancer. Immunity 39: 782-795, 2013.

20. Hänzelmann S, Castelo R and Guinney J: GSVA: Gene set variation analysis for microarray and RNA-seq data. BMC Bioinformatics 14: 7, 2013.

21. Sinicrope FA, Ruan SB, Cleary KR, Stephens LC, Lee JJ and Levin B: bcl-2 and p53 oncoprotein expression during colorectal tumorigenesis. Cancer Res 55: 237-241, 1995

22. Chu PG and Weiss LM: Keratin expression in human tissues and neoplasms. Histopathology 40: 403-439, 2002.

23. Giroux V, Stephan J, Chatterji P, Rhoades B, Wileyto EP, Klein-Szanto AJ, Lengner CJ, Hamilton KE and Rustgi AK: Mouse intestinal Krt15+ crypt cells are radio-resistant and tumor initiating. Stem Cell Reports 10: 1947-1958, 2018.

24. Morris RJ, Liu Y, Marles L, Yang Z, Trempus C, Li S, Lin JS Sawicki JA and Cotsarelis G: Capturing and profiling adult hair follicle stem cells. Nat Biotechnol 22: 411-417, 2004.

25. Depianto D, Kerns ML, Dlugosz AA and Coulombe PA: Keratin 17 promotes epithelial proliferation and tumor growth by polarizing the immune response in skin. Nat Genet 42 : 910-914, 2010

26. Li S, Park H, Trempus CS, Gordon D, Liu Y, Cotsarelis G and Morris RJ: A keratin 15 containing stem cell population from the hair follicle contributes to squamous papilloma development in the mouse. Mol Carcinog 52: 751-759, 2013.

27. Wang GY, Wang J, Mancianti ML and Epstein EH Jr: Basal cell carcinomas arise from hair follicle stem cells in $\mathrm{Ptch}^{+/-}$mice. Cancer Cell 19: 114-124, 2011.

28. Sadraei NH, Shi I, Duan ZH and Shi T: Aberrant signaling pathways in squamous cell lung carcinoma. Clin Cancer Res 18 (3 Suppl): A16, 2012.

29. Tai G, Ranjzad P, Marriage F, Rehman S, Denley H, Dixon J, Mitchell K, Day PJ and Woolf AS: Cytokeratin 15 marks basal epithelia in developing ureters and is upregulated in a subset of urothelial cell carcinomas. PLoS One 8: e81167, 2013.

30. Zhang C, Liang Y, Ma MH, Wu KZ and Dai DQ: KRT15, INHBA MATN3, and AGT are aberrantly methylated and differentially expressed in gastric cancer and associated with prognosis. Pathol Res Pract 215: 893-899, 2019.

31. Evangelista MT and North JP: Comparative analysis of cytokeratin 15, TDAG51, cytokeratin 20 and androgen receptor in sclerosing adnexal neoplasms and variants of basal cell carcinoma. J Cutan Pathol 42: 824-831, 2015.

32. Weisfelner ME and Gottlieb AB: The role of apoptosis in human epidermal keratinocytes. J Drugs Dermatol 2: 385-391, 2003.

33. Elias PM: Stratum corneum defensive functions: An integrated view. J Invest Dermatol 125: 183-200, 2005.

34. Lippens S, Denecker G, Ovaere P, Vandenabeele P and Declercq W: Death penalty for keratinocytes: Apoptosis versus cornification. Cell Death Differ 12 (Suppl 2): S1497-S1508, 2005.

35. Moody CA and Laimins LA: Human papillomavirus oncoproteins: Pathways to transformation. Nat Rev Cancer 10: 550-560, 2010.

36. Yi R, Poy MN, Stoffel M and Fuchs E: A skin microRNA promotes differentiation by repressing 'stemness'. Nature 452 : 225-229, 2008

37. Lane DP: Cancer. p53, guardian of the genome. Nature 358: $15-16,1992$.

38. Kandoth C, McLellan MD, Vandin F, Ye K, Niu B, Lu C, Xie M Zhang Q, McMichael JF, Wyczalkowski MA, et al: Mutational landscape and significance across 12 major cancer types. Nature 502: 333-339, 2013

39. Farhan M, Wang H, Gaur U, Little PJ, Xu J and Zheng W: FOXO signaling pathways as therapeutic targets in cancer. Int $\mathrm{J}$ Biol Sci 13: 815-827, 2017.

40. Napoli $\mathrm{M}$ and Flores ER: The family that eats together stays together: New p53 family transcriptional targets in autophagy. Genes Dev 27: 971-974, 2013.

41. Napoli M and Flores ER: The p53 family orchestrates the regulation of metabolism: Physiological regulation and implications for cancer therapy. Br J Cancer 116: 149-155, 2017.
42. Prokhorova EA, Zamaraev AV, Kopeina GS, Zhivotovsky B and Lavrik IN: Role of the nucleus in apoptosis: Signaling and execution. Cell Mol Life Sci 72: 4593-4612, 2015.

43. Vaughan-Shaw PG, O'Sullivan F, Farrington SM, Theodoratou E, Campbell H, Dunlop MG and Zgaga L: The impact of vitamin D pathway genetic variation and circulating 25-hydroxyvitamin D on cancer outcome: Systematic review and meta-analysis. Br J Cancer 116: 1092-1110, 2017

44. Ferrer-Mayorga G, Gómez-López G, Barbáchano A Fernández-Barral A, Peña C, Pisano DG, Cantero R, Rojo F, Muñoz A and Larriba MJ: Vitamin D receptor expression and associated gene signature in tumour stromal fibroblasts predict clinical outcome in colorectal cancer. Gut 66: 1449-1462, 2017.

45. Carrasco-Garcia E, Moreno M, Moreno-Cugnon L and Matheu A: Increased Arf/p53 activity in stem cells, aging and cancer. Aging Cell 16: 219-225, 2017.

46. Strasser A, O'Connor L and Dixit VM: Apoptosis signaling. Annu Rev Biochem 69: 217-245, 2000

47. Wang Q and Liu J: Regulation and immune function of IL-27. Adv Exp Med Biol 941: 191-211, 2016.

48. Dai YJ, Hu F, Li H, Huang HY, Wang DW and Liang Y: A profiling analysis on the receptor ACE2 expression reveals the potential risk of different type of cancers vulnerable to SARS-CoV-2 infection. Ann Transl Med 8: 481, 2020.

49. Lin TC, Yeh YM, Fan WL, Chang YC, Lin WM, Yang TY and Hsiao M: Ghrelin upregulates oncogenic Aurora A to promote renal cell carcinoma invasion. Cancers (Basel) 11: 303, 2019.

50. Zhang $\mathrm{J}$ and Xie T: Ghrelin inhibits cisplatin-induced MDA-MB-231 breast cancer cell apoptosis via PI3K/Akt/mTOR signaling. Exp Ther Med 19: 1633-1640, 2020.

51. Orange JS: Natural killer cell deficiency. J Allergy Clin Immunol 132: 515-525, 2013

52. Smyth MJ, Crowe NY and Godfrey DI: NK cells and NKT cells collaborate in host protection from methylcholanthrene-induced fibrosarcoma. Int Immunol 13: 459-463, 2001.

53. Smyth MJ, Thia KY, Street SE, Cretney E, Trapani JA, Taniguchi M, Kawano T, Pelikan SB, Crowe NY and Godfrey DI: Differential tumor surveillance by natural killer (NK) and NKT cells. J Exp Med 191: 661-668, 2000.

54. Caligiuri MA: Human natural killer cells. Blood 112: 461-469, 2008.

55. Müller-Hermelink N, Braumüller H, Pichler B, Wieder T, Mailhammer R, Schaak K, Ghoreschi K, Yazdi A, Haubner R, Sander CA, et al: TNFR1 signaling and IFN-gamma signaling determine whether $\mathrm{T}$ cells induce tumor dormancy or promote multistage carcinogenesis. Cancer Cell 13: 507-518, 2008.

56. Böttcher JP, Bonavita E, Chakravarty $\mathrm{P}$, Blees $\mathrm{H}$, Cabeza-Cabrerizo M, Sammicheli S, Rogers NC, Sahai E, Zelenay S and Reis E Sousa C: NK cells stimulate recruitment of $\mathrm{cDC} 1$ into the tumor microenvironment promoting cancer immune control. Cell 172: 1022-1037.e14, 2018.

57. Fannon M, Forsten-Williams K, Dowd CJ, Freedman DA, Folkman $J$ and Nugent MA: Binding inhibition of angiogenic factors by heparan sulfate proteoglycans in aqueous humor: Potential mechanism for maintenance of an avascular environment. FASEB J 17: 902-904, 2003.

58. Gooch JL, Lee AV and Yee D: Interleukin 4 inhibits growth and induces apoptosis in human breast cancer cells. Cancer Res 58: 4199-4205, 1998.

59. Bruno TC: New predictors for immunotherapy responses sharpen our view of the tumour microenvironment. Nature 577: 474-476, 2020.

60. Petitprez F, de Reyniès A, Keung EZ, Chen TW, Sun CM, Calderaro J, Jeng YM, Hsiao LP, Lacroix L, Bougoüin A, et al: $B$ cells are associated with survival and immunotherapy response in sarcoma. Nature 577: 556-560, 2020.

61. Helmink BA, Reddy SM, Gao J, Zhang S, Basar R, Thakur R, Yizhak K, Sade-Feldman M, Blando J, Han G, et al: B cells and tertiary lymphoid structures promote immunotherapy response. Nature 577: 549-555, 2020.

62. Zhu J, Yamane H and Paul WE: Differentiation of effector CD4 T cell populations (*). Annu Rev Immunol 28: 445-489, 2010.

63. Aspord C, Pedroza-Gonzalez A, Gallegos M, Tindle S, Burton EC, Su D, Marches F, Banchereau J and Palucka AK: Breast cancer instructs dendritic cells to prime interleukin 13-secreting $\mathrm{CD}^{+} \mathrm{T}$ cells that facilitate tumor development. J Exp Med 204: 1037-1047, 2007. 
64. He D, Li H, Yusuf N, Elmets CA, Li J, Mountz JD and Xu H: IL-17 promotes tumor development through the induction of tumor promoting microenvironments at tumor sites and myeloid-derived suppressor cells. J Immunol 184: 2281-2288, 2010.

65. Todaro M, Lombardo Y, Francipane MG, Alea MP, Cammareri P, Iovino F, Di Stefano AB, Di Bernardo C, Agrusa A, Condorelli G, et al: Apoptosis resistance in epithelial tumors is mediated by tumor-cell-derived interleukin-4. Cell Death Differ 15: 762-772, 2008.

66. Liu X, Mo W, Ye J, Li L, Zhang Y, Hsueh EC, Hoft DF and Peng G: Regulatory $\mathrm{T}$ cells trigger effector $\mathrm{T}$ cell DNA damage and senescence caused by metabolic competition. Nat Commun 9: 249, 2018.
67. Wang L, Yi T, Kortylewski M, Pardoll DM, Zeng D and Yu H: IL-17 can promote tumor growth through an IL-6-Stat 3 signaling pathway. J Exp Med 206: 1457-1464, 2009.

(i) (9) This work is licensed under a Creative Commons Attribution-NonCommercial-NoDerivatives 4.0 International (CC BY-NC-ND 4.0) License. 